\title{
Save the WHO Program for Hearing Loss Prevention
}

\author{
By the Coalition for Global Hearing Health Advocacy Committee: Sara K. Mamo, AuD; Jean Johnson, DrPH; \\ James Saunders, MD; Jackie Clark, PhD; Susan Emmett, MD; Shehla Afridi, AuD; \& Lady Jean Wilson, OBE
}

The World Health Organization (WHO) is of critical importance to the long-term goals of preventing hearing loss and intervening for those in need. Since establishing the Program for the Prevention of Deafness and Hearing Impairment (PDH) in the mid-1980s, the WHO has been the source of policy statements, guidelines, and prevalence data needed to develop national plans for ear and hearing health.

Currently, the program is in crisis: due to a lack of sufficient external funding, the post at the PDH may be abolished at the end of 2013. However, we believe that without the continued work of the program, the prevention and detection of hearing loss will not become a standard of care in all national health systems, and affected individuals will be denied the basic human right to hear.

\section{THE GLOBAL NEED}

There is an ever-growing number of projects looking for ways to help individuals with hearing loss from the ground up, but, in the long term, we should also be working from the top down.

In 2013, the World Health Organization estimated that 360 million people worldwide had disabling hearing loss (WHO fact sheet on deafness and hearing loss). It is incredible that about half of these cases are preventable.

Preventable causes of hearing loss include chronic otitis media, excessive noise, ototoxic drugs, antenatal and perinatal complications, meningitis, measles, mumps, wax or foreign bodies, and trauma. Given the medical nature of most of these conditions, hearing loss prevention needs to be addressed at the health system level.

Only the WHO has the ability to work with member states to ensure national health plans have basic protections to prevent avoidable hearing loss. At the same time, the PDH is in a position to help develop documents and protocols that can be used at the community health level.

With expanding populations in developing countries, which bear the greatest burden of disabling hearing loss, and a rise in the aging population of industrialized nations, we must have a truly global perspective on the challenges of hearing loss and prevention. The $\mathrm{WHO}$ and, specifically, an able officer at the PDH offer the best prospects for developing global solutions to the tremendous and growing problem of disabling hearing loss.

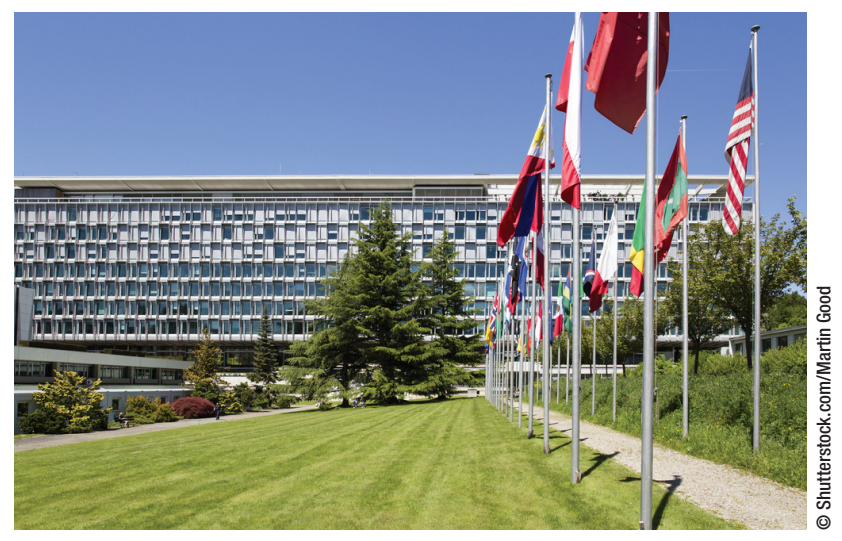

Only the World Health Organization, whose Geneva headquarters are shown here, can work with member states to ensure that national health plans have basic protections to prevent avoidable hearing loss, Dr. Mamo and coauthors say.

\section{PRODUCTIVE AND EFFECTIVE}

Since December 2011, Shelly Chadha, MD, has held the post of program officer for the $\mathrm{PDH}$, and she has been incredibly productive.

One major objective of her post is to meet with and support member states in efforts to integrate primary ear and hearing care into their healthcare policies. Contact has been made with at least 10 nations in the past 18 months. Sri Lanka, Nicaragua, Guinea, Egypt, Malawi, and Mongolia are all developing national plans consistent with their priorities and resources.

Another objective is to raise awareness and establish effective partnerships within the World Health Organization. Discussions have been held with the following programs: Disability and Rehabilitation, Research and Training in Tropical Diseases, Aging and Life Course, Occupational and Environmental Health, Emergency and Essential Surgical Care, Medical Devices, Essential Medicines List, Immunization, and Child Health.

It is easy to see why the prevention of and intervention in hearing loss on a global scale benefit from having a dedicated program officer who is a liaison with these different programs, all of which are critical to ear and hearing health but do not often consider their role in preventing hearing loss.

While it is critical to support the development of national plans that integrate primary ear and hearing care as we move forward, so much more must be done. Specific goals include 
contributing to necessary research on the global epidemiology of hearing loss and its economic impact.

To meet the needs of all people with hearing loss and improve their quality of life, we also have to understand how the effects of hearing loss vary across communities and societies. Again, such global considerations require the leadership of the World Health Organization.

\section{PLEASE ACT NOW!}

Many nongovernmental organizations (NGOs) and privatesector partnerships have more resources and flexibility to be action oriented compared with the $\mathrm{PDH}-\mathrm{a}$ situation that seems to have diminished support for the latter.

However, the PDH's leadership in policy, position papers, and epidemiological data makes the World Health Organization different from and critical to the work of the

\section{The WHO and, specifically,} an able officer at the PDH offer the best prospects for developing global

\section{solutions to the tremendous}

\section{and growing problem}

of disabling hearing loss.

action-oriented groups in reducing the global burden of hearing loss (Am J Public Health 2006;96[1]:62-72).

While many strides have been made in the past 25 years, the prevalence of preventable hearing loss is still inexcusably high. Please join the prestigious international consortium of organizations that provide funding to maintain the post of PDH program officer at the World Health Organization: American Academy of Otolaryngology-Head and Neck Surgery, CBM, Hearing Conservation Council, International
Federation of Oto-Rhino-Laryngological Societies, and International Society of Audiology.

To become a contributing supporter of the Program for the Prevention of Deafness and Hearing Impairment, please visit the Coalition for Global Hearing Health. H

Dr. Mamo is a PhD candidate in the Division of Speech and Hearing Sciences at the University of North Carolina at Chapel Hill. Dr. Johnson is director of the Center on Disability Studies at the University of Hawaii at Manoa. Dr. Saunders is coordinator-elect for international affairs for the American Academy of Otolaryngology_-Head and Neck Surgery and associate professor of otology and neurotology at Dartmouth-Hitchcock Medical Center. Dr. Clark is clinical associate professor at the University of Texas at Dallas and a research scholar at University of the Witwatersrand in Johannesburg, South Africa. Dr. Emmett is a resident in the Department of Otolaryngology-Head and Neck Surgery at Johns Hopkins University School of Medicine and the Department of International Health at Johns Hopkins Bloomberg School of Public Health. Dr. Afridi received her Doctor of Audiology degree in May from the University of Washington in Seattle. Lady Wilson is cofounder and chair of the Hearing Conservation Council, president of the UK IMPACT Foundation, and vice president of Sightsavers International.

\section{Audiology Without Borders}

Our Audiology Without Borders column, featured each month in the $H J$ eNewsletter, highlights humanitarian hearing healthcare programs.

The column is edited by active humanitarians Jackie Clark, PhD, and King Chung, PhD. Dr. Clark is a clinical associate professor at the University of Texas at Dallas and a research scholar at University of the Witwatersrand in Johannesburg; and Dr. Chung is an associate professor of audiology at Northern Illinois University in DeKalb.

Let us know about your humanitarian program! Send the details to HJ@wolterskluwer.com-manuscripts should be about 1,000 words, and photographs are also welcome (300 dpi in jpg, tif, or gif format).

Read past Audiology Without Borders columns in a special collection at http://bit.ly/HJAudWB.

\section{Return to thehearingjournal.com}

